\title{
Reconstruction of residual cleft nasal deformities using a rib cartilage caudal strut graft: Diagnosis and surgical approach
}

\author{
Jeffrey C Posnick* and Brian Kinard \\ Posnick Center for Facial Plastic Surgery, Chevy Chase, USA
}

\begin{abstract}
Importance: The final staged surgery for the teenager born with cleft lip and palate is typically nasal reconstruction. Residual nasal deformities in the adolescent/ adult with a repaired cleft lip and palate is characterized by the under corrected aspects of the malformation not addressed at the time of primary repair, the effects of revision procedures that may have been carried out in childhood, and any restrictive naso-maxillary deformities that occur during growth.

Observations: The rigidity of rib cartilage caudal strut graft, secured with a Kirschner $(\mathrm{K})$ wire through an open approach is often required to provide optimal nasal tip support, projection, and symmetry. The diagnosis of secondary cleft nasal deformities and this surgical approach to reconstruction is reviewed.

Conclusions and relevance: The use of a semi-rigid rib cartilage caudal strut graft secured at the maxillary base by a K-wire placed through an open approach as a component of the cleft lip nasal reconstruction is effective and often necessary. A methodical approach to graft harvesting and recipient site reconstruction optimizes operating room efficiency and best ensures a predictable recovery.
\end{abstract}

\section{Introduction}

Secondary cleft nasal deformities are defined as those that are present despite primary operative maneuvers. Residual nasal deformities in the adolescent/adult with a repaired unilateral cleft lip and palate (UCLP) or bilateral cleft lip and palate (BCLP) are characterized by the under corrected aspects of the malformation not addressed at the time of primary repair, the effects of revision procedures that may have been carried out in childhood, and any restrictive naso-maxillary deformities that occur during growth.

Dibbel was the first to describe the use of an autogenous rib cartilage graft as a caudal strut to correct cleft nasal tip deformities [1]. He accomplished this through a closed rhinoplasty approach and secured the graft to the quadrangular cartilage with sutures. Takato also described use of an autogenous rib cartilage caudal strut graft for BCLP [2]. Like Dibbel [1], he stressed the need for columella soft tissue augmentation with flaps taken from the upper lip and then use of an Abbe flap from the lower lip to reconstruct the deficient upper lip. Hafezi and colleagues, as well as Byrd et al. [3] reported using a rib cartilage graft to correct cleft nasal deformities for both BCLP and UCLP [4]. Mori at al. [5] reported on technique and complications in eight BCLP children and adults undergoing nasal tip reconstruction with autogenous rib cartilage through a closed rhinoplasty.

Through the pioneer work of Gunter, the open approach to cosmetic rhinoplasty has become routine [6]. Gunter also described the use of a rib cartilage caudal strut graft, skewered by a Kirschner wire (K-wire), to prevent warping in secondary rhinoplasty when septal cartilage was not available $[7,8]$. Byrd described an algorithm for the reconstruction of secondary cleft nasal deformities that included the option of rib cartilage grafting but offered only limited information regarding surgical technique [4]. The senior author (JCP) routinely uses a rib cartilage caudal strut graft, secured to the maxillary base by a K-wire, as part of an open rhinoplasty to correct secondary cleft lip nasal deformities. The diagnosis of secondary cleft nasal deformities and the senior author's modified approach for correction with use of a rib cartilage strut graft are detailed.

\section{Evaluation of the nose in the adolescent with a repaired UCLP}

The most favourable definitive rhinoplasty results in the UCLP adolescent are achieved after any underlying maxillary skeleton deformities including irregularities and defects of the floor of the nose, pyriform rims and anterior nasal spine are first addressed. Even when mixed dentition bone grafting has been successful, the need for orthognathic surgery is frequent and should be addressed prior to definitive rhinoplasty $[9,10]$. Correction of the presenting secondary nasal deformities in the UCLP adolescent requires consideration of:

- Maxillary hypoplasia in relation to the mandible below and the facial skeleton above.

- Hypoplasia and asymmetries of the pyriform rims, nasal floor, and anterior nasal spine.

*Correspondence to: Jeffrey C Posnick, Posnick Center for Facial Plastic Surgery, Chevy Chase, USA, Tel: 301-986-9475; Fax: 301-986-1974; E-mail: jposnick@drposnick.com

Key words: restrictive naso-maxillary deformities, cleft lip nasal reconstruction, graft harvesting

Received: June 03, 2019; Accepted: June 21, 2019; Published: June 25, 2019 
- Deformity of the osseous nasal vault (dorsal hump, asymmetries, or widening).

- Flattening and twisting of the cartilage vault of the nose.

- Limited projection and asymmetries of the nasal lobule and tip (cleft side flattening).

- The ipsilateral alar rim may lie caudal, lateral, and posterior as a reflection of a deformed and deficient lower lateral cartilage.

- Scarring and hypoplasia of the overlying nasal soft tissue envelope.

- Scarring of the internal nasal lining (vestibular web on cleft side).

- Deviations of the nasal septum causing airway obstruction and sinus blockage.

- Hypertrophy of the inferior turbinate(s) causing airway obstruction and sinus blockage.

\section{Basic UCLP rhinoplasty considerations}

In most adolescents/adults with a repaired UCLP, an adequate overlying soft tissue envelope and underlying internal nasal lining is present without need for soft tissue augmentation. While the nasal bones may be deformed, they are usually with enough structure to allow reconstruction through a combination of osteotomies (straightening and in fracture) and dorsal hump reduction. The mid-cartilaginous nasal vault may be crooked and/or flat to the extent that spreader grafts are required. An autogenous cartilage caudal strut graft will be needed for tip straightening and projection in virtually all cases. In some cases, septal cartilage will be of adequate strength to be used as a caudal strut graft. If greater structural support is required to elevate and straighten the nasal tip, use of a rib cartilage caudal strut graft is preferred. If the quality of the lower lateral cartilages (LLC's) is adequate, reconstruction by reduction of cephalic excess and suture-pexy of each LLC to the other and then over the top of the caudal strut graft is carried out. If the cleft side LLC in particular is significantly hypoplastic, then an augmentation cartilage graft(s) is required (i.e. alar contour graft or lateral crural strut graft).

\section{Evaluation of the nose in an adolescent with a repaired BCLP}

As with UCLP, nasal aesthetics in the BCLP adolescents/adults requires a reconstructed underlying skeletal base (i.e. floor of the nose, pyriform rims, and anterior nasal spine). We know that a majority of repaired BCLP adolescents/adults will have naso-maxillary deficiency to the extent that orthognathic surgery is required $[9,10]$. If so, the cleft jaw deformity should be corrected prior to definitive rhinoplasty. Residual BCLP nasal deformities in the adolescent/adult may include:

- Short columella (hypoplasia of the overlying soft tissues) with limited nasal tip projection.

- Lack of caudal projection of the anterior aspect of the quadrangular septal cartilage.

- Flattening of the osseo-cartilaginous nasal vault.

- Nasal tip bifidity (separation of the LLC's).

- Lateral displacement of the alar base(s) with horizontal rotation of the nostrils due to buckling and downward rotation of the LLC.

- Scarring of the internal nasal lining (vestibular webbing).

- Deviations of the nasal septum causing airway obstruction and sinus blockage.
- Hypertrophy of the inferior turbinates causing airway obstruction and sinus blockage.

\section{Basic BCLP rhinoplasty considerations}

A relatively symmetric nose with a short columella and flat nasal tip typically defines the adolescent BCLP nasal deformity. A fundamental nasal aesthetic deformity that requires reconstruction is the poor tip projection and shape. This deficiency can generally be reconstructed with use of a crafted autogenous cartilage caudal strut graft. A rib cartilage graft provides the rigidity typically required to stretch the nasal soft tissue envelope over the graft without bending (warping) to achieve needed tip projection. Stretching of the nasal soft tissue envelope over a semi-rigid rib cartilage caudal strut graft has eliminated the need for forked (soft tissue) flaps taken from the upper lip. The LLC's are also modified (see UCLP) and then sutured together either over the top of the graft if feasible or if not then adjacent to the top of the rib cartilage caudal strut graft. The top of the caudal strut graft forms the new nasal tip. If significant LLC hypoplasia and deformity exists, then augmentation cartilage grafts are required (see UCLP).

\section{Timing of definitive cleft nasal reconstruction}

The definitive cleft lip rhinoplasty is best undertaken after successful mixed dentition alveolar bone grafting, including stabilization of the pre-maxilla in the case of BCLP and then successful orthognathic surgery if required.

\section{Reconstruction of cleft nasal deformities in the adolescent/ adult: step by step approach (Figure 1 and Figure 2)}

- General anaesthesia through orotracheal intubation (Figure 1 and Figure 2). The endotracheal tube is secured to the midline of the chin region with tape.

- Local anaesthesia is injected for haemostasis below the soft tissue envelope of the nose.

- Stair-step columella (skin) and bilateral marginal (intra-nasal) incisions are completed.

- The overlying cutaneous soft tissue envelope is elevated up the columella, superficial to the LLC's and upper lateral cartilages (ULC's), subperiosteal over the nasal bones to the radix and laterally towards the maxillary process on each side. At wound closure, the fully elevated soft tissue envelope will be advanced caudally and stretched over the reconstructed cartilaginous vault and nasal tip.

- If an osseous vault dorsal hump requires reduction it is completed with rasps.

- If a cartilage vault dorsal hump requires reduction it is completed with a sharp knife after separating the quadrangular septal cartilage from its attachment to the ULC's.

- The medial crura of the LLC's are separated from each other using scissors dissection down to the base of the maxilla/anterior nasal spine.

- The caudal aspect of the quadrangular cartilage is resected if needed to avoid buckling later in the reconstruction when the caudal strut graft is placed.

- If a submucosal resection of the cartilaginous septum is required to improve nasal airflow; it is initiated using a knife. The sharp knife incisions are located to maintain approximately $10 \mathrm{~mm}$ of caudal and $10 \mathrm{~mm}$ of dorsal septal cartilage support for the nose. The swivel knife is then inserted and used to excise the posterior located deviated/buckled septal cartilage (submucous resection). A rongeur 
is used to remove any buckled/deviated portions of vomer and perpendicular plate of ethmoid that are obstructing nasal airflow.

- If the inferior turbinates are hypertrophic and require reduction to improve nasal airflow, it is accomplished by one of several methods including: scissors resection of the inferior aspect of each turbinate; out fracture; submucosal (intramural) removal; or submucosal cauterization.

- The rib cartilage graft will have been harvested prior to the nasal surgery. The caudal strut graft is crafted from the harvested rib cartilage. (The potential for graft warping is related to the inherent structure of costal cartilage which may lead to curvature of the caudal strut [14-17]. Insertion of a Kirschner wire through the spine of the graft will prevent warping but results in an unnaturally stiff nose [7]. The complication of caudal strut graft warping is greatly reduced by creating a crafted graft from the central block of cartilage that is carved in balanced cross sections [15-17]. If warping of the carved graft is going to occur, it is likely to do so within 30 minutes of harvesting/crafting [7]. In the uncommon case that warping is observed, a separate 35 threaded $\mathrm{K}$-wire is skewered through the spine of the graft prior to placement at the base of the maxilla [1,7]).

- The crafted cartilage caudal strut graft extends from the base of the maxilla (anterior nasal spine region) and then up to form the new nasal tip. At completion of rhinoplasty the graft length will typically vary in length from $28-34 \mathrm{~mm}$. The graft is secured to the base of the maxilla with a $\mathrm{K}$-wire (0.035-in threaded) which is drilled into the anterior nasal spine region for a depth of approximately 5 millimetres (Figure 3). Placement of the K-wire is between the central incisors and just below the floor of the nose. Typically, a rongeur is used to flatten the base where the K-wire will be inserted. If the anterior nasal spine is present, it is also reduced with a rongeur for placement of the K-wire. The inserted $\mathrm{K}$-wire is cut leaving an extension of 3-4 millimetres above the exposed base of the maxilla. The caudal strut graft is then pierced into the K-wire to prevent movement of the graft at the maxillary base during the healing process. The direction (angle) of insertion of the graft will control the nasal tip location and influence the nasolabial angle. The height of the caudal strut
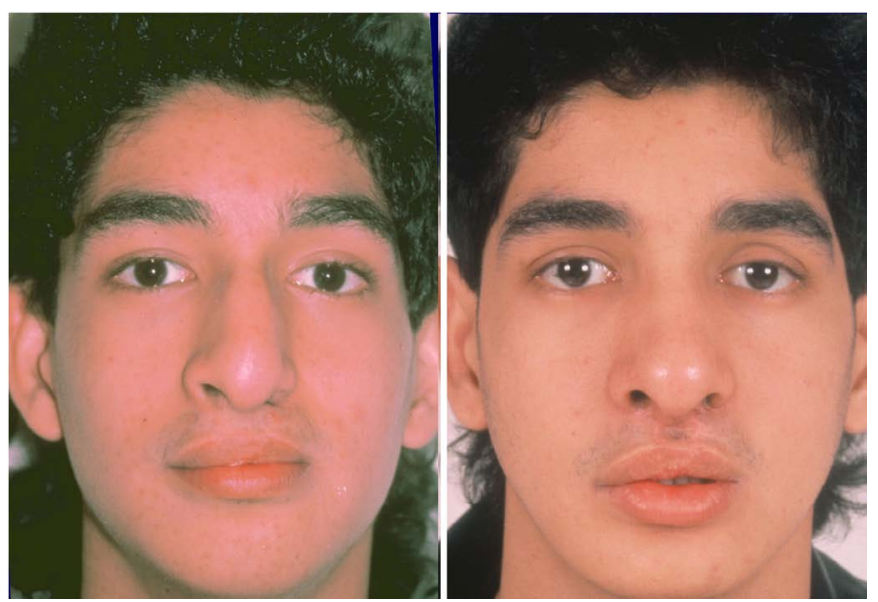

Figure 1. This is a Hispanic male born with complete BCLP. He underwent lip and palate repair and ineffective bone grafting in the mixed dentition by another surgeon. He presented to the primary investigator (JCP) in 1987 at 15 years of age with residual naso-maxillary deformities. He underwent cleft orthognathic surgery and six months later definitive cleft nasal reconstruction. The open rhinoplasty included: nasal osteotomies (in-fracture and straightening); minimal dorsal reduction (bone and cartilage); modification of LLC's (excision of cephalic excess) and rib cartilage caudal strut grafting as described in the text. Frontal views before and after reconstruction [11].


Figure 2. This is a Caucasian male born with complete UCLP. He was treated by another surgeon including primary cleft lip and palate repair, and then cleft lip, nasal tip and palate revisions in childhood including placement of a pharyngeal flap. This was followed by ineffective mixed dentition bone grafting and failed Le Fort I osteotomy with an external (DO) device. He was referred to the primary investigator (JCP) at 17 years of age with residual naso-maxillary deformities. He underwent successful redo cleft orthognathic surgery. One year later, he underwent definitive cleft nasal reconstruction through an open approach. This included: nasal osteotomies (in-fracture and straightening); dorsal reduction (bone and cartilage); modification of LLC's (excision of cephalic excess) and rib cartilage caudal strut grafting as described in the text. Oblique facial views before and after nasomaxillary reconstruction [12].

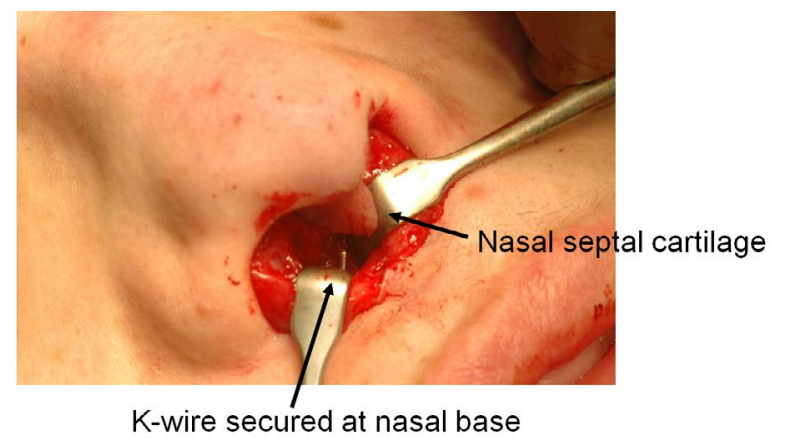

projecting up to pierce \& secure strut graft

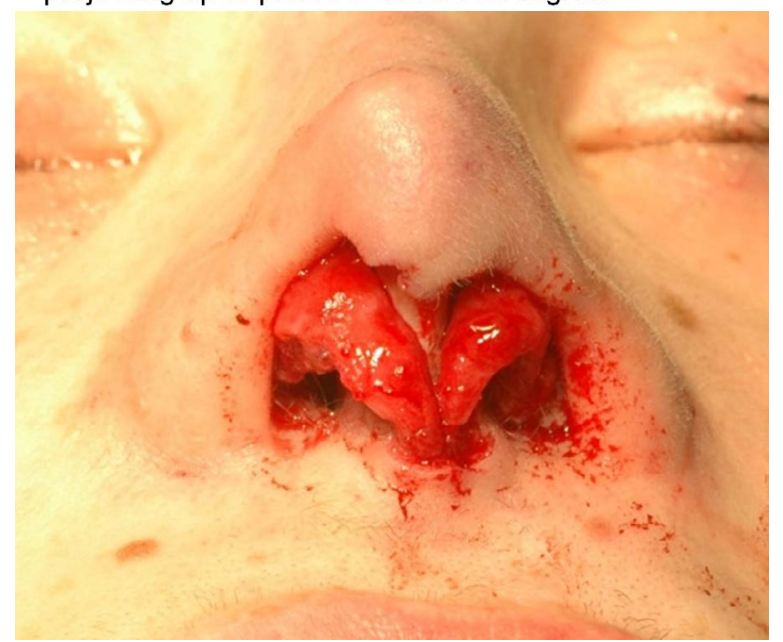

Figure 3. Intra-operative views of rib cartilage graft harvesting and crafting. A) Through an open rhinoplasty approach, the base of the anterior maxilla is exposed. A K-wire is inserted through the anterior maxilla $(4 \mathrm{~mm})$ and left to project out of the bone $(4 \mathrm{~mm})$. The caudal aspect of the septal cartilage is shown in relationship to the projecting K-wire. The caudal aspect of the quadrangular cartilage will be cut back to prevent buckling against the graft. B) The soft tissue envelope of the nose is temporarily draped over the caudal strut graft to judge the preferred height of the graft. This is prior to suturing the LLC's over the top of the graft and then finally redraping and suturing the soft tissue envelope in place [13]. 
graft is refined (cut back) as needed after redraping of the soft tissue envelope and confirming optimal nasal aesthetics.

- The lower lateral cartilages, having been trimmed of any cephalic excess, are sutured together over the top of caudal strut graft to form the most superficial aspect of the nasal tip.

- If spreader grafts are required to reconstruct the cartilage vault, they are crafted from harvested rib cartilage and sutured in place.

- If the LLC(s) require augmentation/reconstruction, the needed graft is crafted from the harvested rib cartilage. The LLC cartilage graft on each side is on layed over the native LLC and secured (laminated) with suture. The graft will stabilize the external nasal valve and the medial crus are joined (by suture) in front of the strut graft to limit widening of the columella.

- The stair-step columella (skin) incision is initially closed with one deep layer stitch. Skin layer closure is then accomplished. The rim incisions are closed with resorbable sutures.

- Antibiotic ointment is injected into the nasal cavity. Packing in each nostril is carried out to decrease intra nasal swelling/bleeding and to splint the nasal bones (osteotomies) in place.

- Steri-Strips are placed over the dorsal skin.

- A custom splint is conformed over the dorsum.

- The packs are removed within 48 hours.

- The dorsal splint and sutures are removed 5 to 7 days postoperatively.

\section{Harvesting rib cartilage graft (donor site): Step-by-step approach}

- Orotracheal intubation as required for the rhinoplasty procedure (taped to the chin).

- The specific rib cartilage to be harvested is palpated through the right anterior chest wall below the infra-mammary fold and close to the junction of the sternum. The planned incision (i.e. $\sim 4 \mathrm{~cm}$ ) is marked directly over the rib cartilage to be harvested.

- Betadine solution is used for the preparation of the right anterior chest wall and the head and neck.

- Local anaesthesia is injected into the soft tissues below the planned incision and to the depth of the rib cartilage to be harvested.

- With the use of a knife, and then cautery, the incision is carried through the skin, the subcutaneous tissue, the fascia, and the rectus abdominus muscle directly over the rib cartilage to be harvested.

- The perichondrium is scored with a knife directly over the mid portion of the long axis of the rib cartilage to be harvested.

- Curved and straight elevators dissect in the subperichondrial plane circumferentially around the cartilage to be harvested.

- A curved subperichondrial dissector is inserted underneath and around the rib cartilage to confirm circumference exposure in the subperichondrial plane for the length of the graft to be harvested.

- A retractor is placed directly underneath the medial location for the incising of the cartilage graft. A knife is then used to incise the cartilage full thickness.

- The retractor is then repositioned just below the other end of the cartilage at the location for incision. The knife is used to incise the cartilage full thickness.
- The cartilage graft ( $3.5-4 \mathrm{~cm}$ in length) is removed.

- The wound is checked for hemostasis and any exposure into the lung cavity. This is done by filling the wound with saline and the anesthesiologist introduces maximum end tidal volume and holds the area under pressure with the Valsalva maneuver.

- With confirmation of no pneumothorax, wound closure is accomplished. A) The perichondrial sleeve is approximated with interrupted suture ties. $B$ ) The deep and superficial fascia layers are closed with interrupted suture ties. C) Subdermal closure is with interrupted suture ties. D) The skin is closed with subcuticular running suture.

- A limited number of Steri-Strips are placed directly over the closed wound.

- A small piece of cut gauze is placed directly over the Steri-Strips.

- An occlusive dressing is placed over the skin.

- The dressing remains intact for approximately 7 days.

- When the dressing is removed, no additional bandages are placed.

\section{Conclusions}

Definitive cleft nasal reconstruction in the adolescent born with UCLP and BCLP is typically required as part of a staged correction of the malformation. The use of a semi-rigid rib cartilage caudal strut graft secured at the maxillary base by a K-wire placed through an open approach as a component of the rhinoplasty is effective. A methodical approach to graft harvesting and recipient site reconstruction optimizes operating room efficiency and best ensures a predictable convalescence.

\section{References}

1. Dibbell DG (1976) A cartilaginous columellar strut in cleft lip rhinoplasties. Br J Plast Surg 29: 247-250. [Crossref]

2. Takato T, Yonehara Y, Susami T (1995) Columella lengthening using a cartilage graft in the bilateral cleft lip-associated nose: choice of cartilage according to age. $J$ Oral Maxillofac Surg 53: 149-157. [Crossref]

3. Hafezi F, Naghibzadeh B, Ashtiani AK, Mousavi SJ, Nouhi AH, et al. (2013) Correction of cleft lip nose deformity with rib cartilage. Aesthet Surg J 33: 662-673. [Crossref]

4. Byrd HS, El-Musa KA, Yazdani A (2007) Definitive repair of the unilateral cleft lip nasal deformity. Plast Reconstr Surg 120: 1348-1356. [Crossref]

5. Mori Y, Takato T, Hoshi K, Kanno Y, Sugiyama M, et al. (2014) Correction of upturned nasal tip with a costal cartilage graft in bilateral cleft lip patients. J Craniofac Surg 25: e443-e445. [Crossref]

6. Gunter JP, Rohrich RJ (1987) External approach for secondary rhinoplasty. Plast Reconstr Surg 80: 161-174. [Crossref]

7. Gunter JP, Clark CP, Friedman RM (1997) Internal stabilization of autogenous rib cartilage grafts in rhinoplasty: a barrier to cartilage warping. Plast Reconstr Surg 100: 161-169. [Crossref]

8. Gunter JP, Cochran CS, Marin VP (2008) Dorsal augmentation with autogenous rib cartilage. Semin Plast Surg 22: 74-88. [Crossref]

9. Good PM, Mulliken JB, Padwa BL (2007) Frequency of Le Fort I osteotomy after repaired cleft lip and palate or cleft palate. Cleft Palate Craniofac J 44: 396-401. [Crossref]

10. Daskalogiannakis J, Mehta M (2009) The need for orthognathic surgery in patients with repaired complete unilateral and complete bilateral cleft lip and palate. Cleft Palate Craniofac J 46: 498-502. [Crossref]

11. Posnick JC (2014) Aesthetic alteration of the nose: Evaluation and surgery. Orthognathic Surgery Principles and Practice. St Louis: Elsevier. p: 1613-1702.

12. Posnick JC (2014) Grafts Frequently Used During Orthognathic Surgery and for Adjunctive Procedures. Orthognathic Surgery Principles and Practice. St Louis: Elsevier. p: 607-639.

13. Posnick JC (2014) Video 14: Cleft orthognathic surgery for the BCLP deformity [Video file]. Orthognathic Surgery: Principles and Practice. St. Louis: Elsevier. 
Posnick JC (2019) Reconstruction of residual cleft nasal deformities using a rib cartilage caudal strut graft: Diagnosis and surgical approach

14. Marin VP, Landecker A, Gunter JP (2008) Harvesting rib cartilage grafts for secondary rhinoplasty. Plast Reconstr Surg 121: 1442-1448. [Crossref]

15. Gibson T, Davis WB (1958) The distortion of autogenous cartilage grafts: Its cause and prevention. Br J Plast Surg 10: 257-274.
16. Lopez MA, Shah AR, Westine JG, O'Grady K, Toriumi DM (2007) Analysis of the physical properties of costal cartilage in a porcine model. Arch Facial Plast Surg 1: 35-39. [Crossref]

17. Lee MR, Inman J, Ducic Y (2011) Central Segment harvest of costal cartilage harvest in rhinoplasty. Laryngoscope 121: 2155-2158. [Crossref]

Copyright: (C2019 Posnick JC. This is an open-access article distributed under the terms of the Creative Commons Attribution License, which permits unrestricted use, distribution, and reproduction in any medium, provided the original author and source are credited. 